\title{
Obituary
}

\section{Professor G. D. Preston}

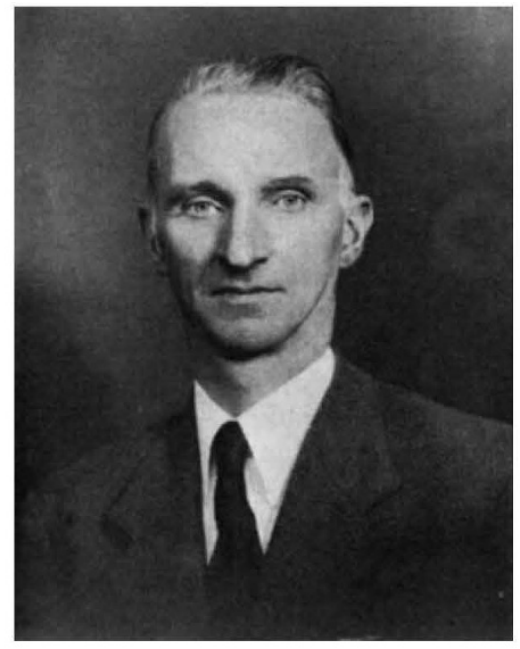

Professor G. D. Preston died at Meigle Cottage Hospital, Perthshire, on June 22, 1972. He was 75.

George Dawson Preston was born in 1896, the son of Professor Thomas Preston, FRS, and was educated at Oundle and Caius College, Cambridge. His school and university careers were separated by service in World War I with the 7th $\mathrm{Bn}$, Alexandra Princess of Wales' Own Yorkshire Regiment, service which was ended by a severe leg wound, the effects of which he suffered with admirable fortitude all his life.

After Cambridge, in 1921, he joined the staff of the Metallurgy Division of the National Physical Laboratory, and so began 22 years of fruitful and pioneering work. The techniques of $\mathrm{X}$-ray and electron diffraction were still in their infancy, and he began a systematic application of them to the crystal structures of metals and alloys, working initially with E. A. Owen and later with L. L. Bircumshaw. During his investigations into the structure of alloys, he formulated the idea that the different atoms show clustering or segregation effects (the so-called Guinier-Preston zones) and it was during his detailed study of Laue photographs of aluminium alloys under different cold working and heat treatment conditions that his attention was drawn to the remarkable background scattering on the plates; not just a general diffuse background, but concentrated regions of intensity part of which at least he found to be strongly temperature dependent. The situation was an interesting one. X-ray methods by the beginning of World War II were being extensively used on a wide variety of problems using the Laue or Bragg reflexions. The diffuse background present in all X-ray photographs was regarded as a nuisance to be minimized by careful experimental technique, although the brilliant theoretical work of Waller had shown what a wealth of valuable information lay in that very background. Unfortunately, Waller's work was not widely known by experimentalists, and, even if known, was not readily interpretable because of its mathematical complexity.

Preston's work sparked off an upsurge of interest in Britain in thermal diffuse scattering, although the principal credit for opening up the field must certainly go to Laval in France, who began a careful investigation into the scattering from various crystals in 1938. Laval's main results, however, were published in such an obscure journal that there can be no doubt that Preston was entirely unaware of his work. In a very short space of time, different groups of workers in Britain, India and the United States all began looking more closely into the phenomenon, although communication difficulties due to wartime conditions meant that they were essentially working independently of each other. Stimulated by the experimental work, various workers redeveloped the fundamental theory, both to simplify the mathematics and to bring out the physical significance. Preston himself gave a qualitative explanation of the effect by assuming that small groups of segregated atoms in the crystal could scatter X-rays independently of the rest of the lattice, and this "geometrical" theory was further developed by W. $\mathbf{H}$. Bragg. The assumption of small independent groups of atoms, however, is hardly reconcilable with our knowledge of the dynamics of crystal lattices, and Max Born showed that the partial agreement with experiment of the simple Preston-Bragg theory was accidental, and that the full Waller theory was necessary for a complete explanation. This work on thermal diffuse scattering was the culmination of Preston's work at the NPL, much of which showed considerable experimental skill. Mounted prints of some of his Laue photographs still elicit admiring comments from workers in the field.

In 1943 Preston left the NPL on his appointment to the Harris chair of physics in University College, Dundee, in the University of St Andrews. During his first few years there, he followed the Scottish tradition of teaching the entire first-year course himself, in addition to giving a normal quota of honours lectures. One curious feature of his teaching will be remembered ruefully by his former students: he had a predilection for quaternion methods, and resolutely refused to use the Gibbs notation, a refusal which he defended with great wit and persuasiveness. With a teaching load like that, and with the ludicrously small research budget at his disposal, it would not have been surprising if he had given up any idea of continuing with his research work. But in fact X-ray equipment was somehow created from government-surplus junk and work on diffuse scattering was continued. In addition, staff were appointed in other fields of X-ray crystallography, and such work has continued in Dundee to this day. In 1944 he was elected to a Fellowship of the Royal Society of Edinburgh. His tenure of the chair in Dundee saw the beginning and very nearly the end of the long, sometimes bitter, negotiations between St Andrews and Dundee on the future of their relationship. $\mathrm{He}$ had little taste for academic intrigue and political bickering, and his dignified and sane attitude throughout the troubled period won him the respect of all his colleagues. $\mathrm{He}$ retired in 1966, just one year before St Andrews and Dundee finally separated with the creation of the new University of Dundee, and it was as Professor Emeritus of that institution that he died.

G. D. Preston was a man of wide culture and gentle character, and he will be sadly missed. $\mathrm{He}$ is survived by his wife, two sons and two daughters.

\section{Announcements}

\section{University News}

Dr W. D. Biggs has been appointed to the newly created professorship of building technology in the Faculty of Urban and Regional Studies at the University of Reading.

Professor David Johns has been appointed head of the department of transport technology, Dr Norman Ashford to a chair in transport planning, and Dr Geoffrey Gregory to a chair in management sciences at the University of Loughborough.

Mr Brian McCloskey has been appointed to the chair of architecture at the Queen's University of Belfast.

Dr D. J. Bartholomew has been appointed to the chair of statistics at the London School of Economics and Political Science.

Dr M. E. H. Turner-Warwick has been appointed to the chair of medicine at the Cardiothoracic Institute, University of London. 